The Astrophysical Journal, 175:L137-L139, 1972 August 1

(C) 1972. The American Astronomical Society. All rights reserved. Printed in U.S.A.

\title{
ON THE OPTICAL SEARCH FOR CENTAURUS X-3
}

\author{
R. J. Brucato, J. Kristian, and J. A. WestPhal \\ Las Campanas Observatory and Hale Observatories, Carnegie Institution \\ of Washington, California Institute of Technology \\ Received 1972 June 5
}

\section{ABSTRACT}

The optical eclipsing binary LR Cen has been eliminated as a candidate for Cen X-3 on the basis of a real discrepancy of orbital periods. We believe that the position coincidence of Wray 795 with Cen $\mathrm{X}-3$ is not statistically significant.

\section{LR CENTAURI}

Among the more spectacular discoveries made by the Uhuru $\mathrm{X}$-ray satellite is the nature of Cen X-3 (=2U 1119-60) which, from the X-ray data alone, has been found to be a member of an eclipsing binary, with most of the $\mathrm{X}$-ray emission in 4.8 pulsations (Schreier et al. 1972). The nearby optical eclipsing binary LR Cen was suggested as a possible candidate for the source by Kristian, Brucato, and Westphal (1972) and by Shklovsky, Cherepaschuk, and Efremov (1972) primarily on the basis of its period, which is within 0.5 percent of that of Cen X-3. The difference in periods is small if accidental but large in comparison with the observational errors and would require a decrease of 0.01 in the period of LR Cen since 1927. However, minima of LR Cen were observed on the following dates this year: JD 2441364.870 (Hiltner 1972), JD 2441383.69 $( \pm 0.04)$, JD 2441394.207 (Peterson 1972), and JD 2441427.741( \pm 0.003$)$. These observations give a period of $2.0957 \pm 0.0002$, compared with $2.08712 \pm 0.00004$ for Cen $\mathrm{X}-3$, and definitely eliminate LR Cen as a candidate. Furthermore, the most recent position of the X-ray source (Murray, private communication) is greatly improved and independently rules out LR Cen as a candidate.

Note that from the variable-star survey in which LR Cen was found (Uitterdijk 1932 and references therein) the $a$ priori probability of finding an eclipsing binary with period and position in as good agreement is $\sim 10^{-4}$. This may have something to say about the general validity of $a$ posteriori calculations of the probability of a particular observed event.

\section{WRAY 795}

Margon and Wray (1972) have suggested the emission-line star Wray 795 as the X-ray source on the basis of position agreement and spectroscopic characteristics. We have obtained spectrograms of this star on 1972 March $9\left(270 \AA \mathrm{mm}^{-1}\right)$ and on 1972 April 8, $16,19\left(60 \AA \mathrm{mm}^{-1}\right)$, using the image-tube spectrograph attached to the Las Campanas 40 -inch (1-m) telescope. The low-dispersion plate shows emission at $\mathrm{H} \alpha$ and $\mathrm{H} \beta$ with the remaining members of the Balmer series in absorption. At the higher dispersion, the $\mathrm{H} \beta$ emission is seen to be superposed on a broad absorption feature. The higher members of the Balmer series are again in absorption, as are a number of $\mathrm{He} \mathrm{I}$ lines. We see no variations in the spectrum on our plates. We estimate the spectral type of Wray 795 to be $\sim$ B5e with $v \sin i \sim 400-450 \mathrm{~km} \mathrm{~s}^{-1}$.

From the distribution of nearby Wray emission-line objects, Margon and Wray (1972) find a probability of 0.03 that the coincidence of Wray 795 with the Cen X-3 error box is accidental. On the basis of a larger sample, we believe that the association is not statistically significant. Since the Wray catalog is unpublished, we have cross-

\section{L137}


correlated Wackerling's (1971) compilation for $7^{\mathrm{h}} \leq \alpha \leq 18^{\mathrm{h}}$ (where a majority of the objects are from Wray's catalog) with the X-ray source positions with $|b|<20^{\circ}$ listed in the 2U catalog (Giacconi et al. 1972). For those sources with error boxes less than about 1 square degree, we found 16 coincidences, compared with 13.5 expected on the basis of chance. For the complete Wackerling catalog, we found 22 coincidences, compared with 22 expected.

\section{GENERAL REMARKS}

The case of Cen X-3 demonstrates with particular clarity the current impasse in the optical identification of X-ray sources. Except for a number of young supernova remnants, the only identified galactic sources are Sco X-1 (near certain), Cyg X-2 (very likely), and Cyg X-1 (possible), and these still are based primarily on positional agreement. In the absence of very accurate X-ray positions $\left(\sim 10^{\prime \prime}\right)$ or theoretical predictions of the optical appearance of X-ray sources, identifications must be based on similarities to known sources and/or the correlation of events (pulsation, flares, binary motions) in both the optical and X-ray regions. But there has been a conspicuous absence of Sco $\mathrm{X}-1$-like objects, and the suggested identifications have been based on "peculiarity" according to ill-defined $a$ posteriori criteria which change from source to source. It seems clear that none of the many suggested identifications can be considered valid until direct evidence is found linking them with the $\mathrm{X}$-ray sources. The binary sources such as Cen X-3 are particularly interesting in this regard, since they involve a parameter, the period, which can be accurately and unambiguously measured. Even the suggested identification of $+34^{\circ} 3815$ with Cyg X-1, for example (Webster and Murdin 1972; Bolton 1972), is not secure. There is no doubt, from the agreement of very accurate positions, that it is the radio source discovered by Hjellming and Wade (1971) and Braes and Miley (1972). But it is becoming increasingly clear that there is a relatively high surface density of faint radio sources in the plane, and there is no good evidence linking either $+34^{\circ} 3815$ or the radio source with Cyg X-1. A determination of activity in the $\mathrm{X}$-ray source which is correlated with the $5^{\text {d }} 6$ period of $+34^{\circ} 3815$ would be irrefutable.

In the case of Cen X-3, the elimination of LR Cen as a candidate leaves Wray 795 as the leading candidate by default, but the evidence either for or against it is not strong. Its positional agreement may easily be accidental, and it appears to be a normal Be star (as opposed to the more unusual object X Per), which as a class do not seem to be associated with X-ray sources.

Using the mass function of Schreier et al. (1972) and an assumed interstellar absorption of no more than 2.5 mag (Neckel 1967), we estimate the maximum apparent magnitude of the Cen X-3 primary as $\sim 11$ mag. There are five stars brighter than $\sim 12$ mag within the $1.5 \sigma$ box. These are shown in figure 1 (plate L2) along with a number of other stars for which we have low-dispersion spectra. Except for Wray 795, none show emission at $\mathrm{H} \alpha$. We believe, however, that all of these stars should be considered as optical candidates for Cen X-3.

We would like to express our appreciation to Mr. Howard Lanning for his assistance in obtaining some of the observational material presented here, to Dr. J. P. Swings for obtaining a number of spectrograms during his observing run at Las Campanas, and to American Science and Engineering, Inc., for providing information on this source in advance of publication. This work was supported in part by the National Aeronautics and Space Administration under grant NGR 09-140-009 to the Carnegie Institution of Washington.

\section{REFERENCES}

Bolton, C. T. 1972, Nature, 235, 271.

Braes, L. L. E., and Miley, G. K. 1972, Nature Phys. Sci., 235, 147.

Giacconi, R., Murray, S., Gursky, H., Kellogg, E., Schreier, E., Tananbaum, H. 1972, Ap. J. (in press). Hiltner, W. 1972, IAU Circ., No. 2398. 


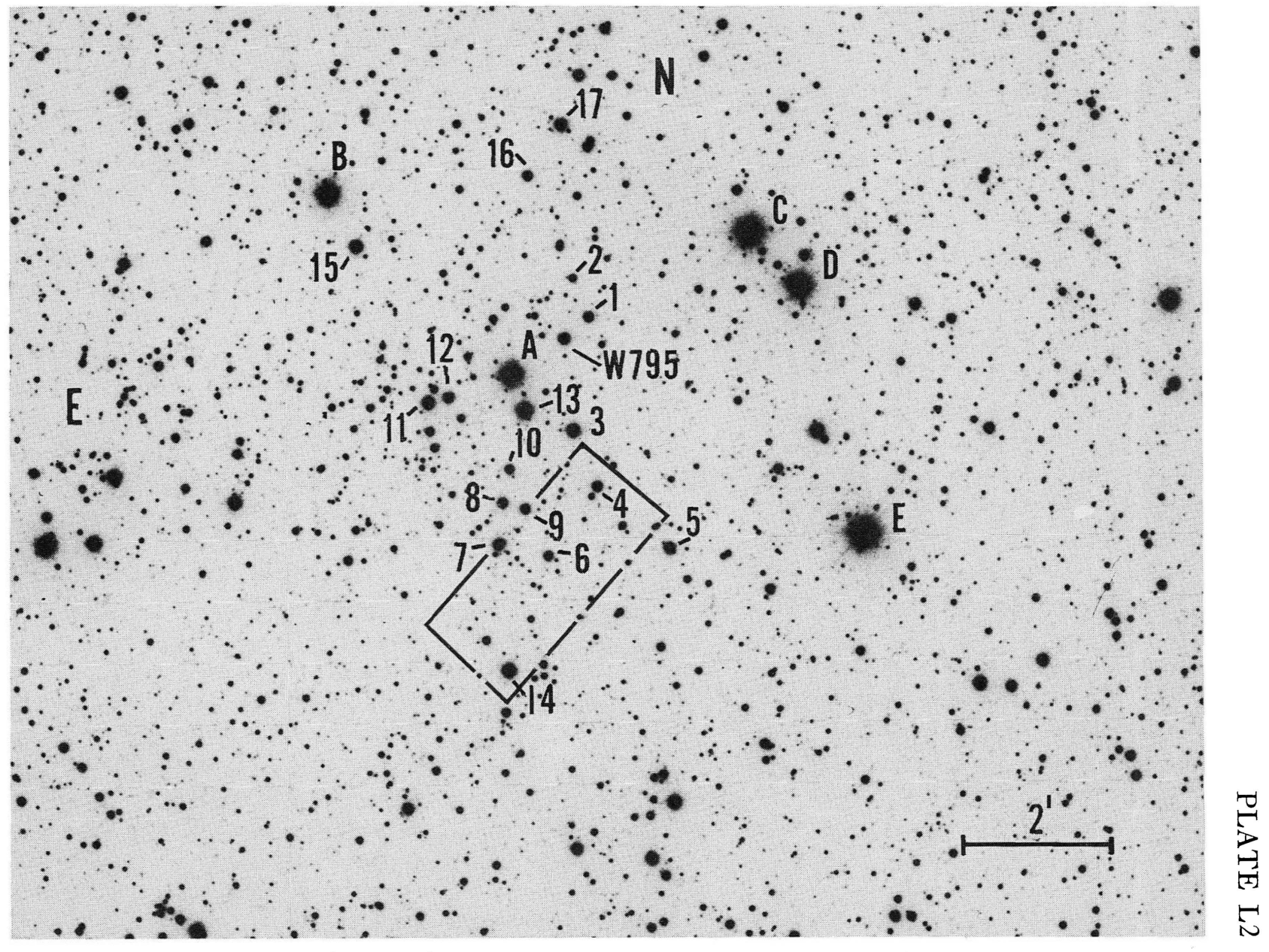

Fig 1 - Cen X-3. The box shown is the $1.5 \sigma$ position from Uhuru measurements (Murray, private communication). Low-dispersion $\left(\sim 270 \AA \mathrm{mm}^{-1}\right)$ spectra of the numbered stars show no emission at Ho. Medium-dispersion ( $60 \AA \mathrm{mm}^{-1}$ ) spectra of the lettered stars show no emission at $\mathrm{H} \beta$. See text for discussion of W795. Identification of the bright stars are: $\mathrm{A}=\mathrm{HD} 306373$ (B8); $\mathrm{B}=\mathrm{HD} 98816$ (A3); $\mathrm{C}=\mathrm{HD} 98724$ (F5); D = HD 98707 (A0); $\mathrm{E}=\mathrm{HD} 98693(\mathrm{A0}) ; 13=\mathrm{CPD}-59^{\circ} 3378 ; 14=\mathrm{CPD}-59^{\circ} 3380$. Spectral types where shown are from the HD catalog.

Brucato, Kristian, and WestPhal (see page L138) 
Hjellming, R. M., and Wade, C. M. 1971, Ap. J. (Letters), 168, L21.

Kristian, J., Brucato, R. J., and Westphal, J. S. 1972, I AU Circ., No. 2395.

Margon, B., and Wray, J. D. 1972, Ap.J. (Letters) (in press).

Neckel, T. 1967, Heidelberg Veröff. Vol. 19.

III. Peterson, B. A. 1972, Nature, 236, 449.

Schreier, E., Levinson, R., Gursky, H., Kellogg, E., Tananbaum, H., and Giacconi, R. 1972, Ap. J. (Letters), 172, L79.

Shklovsky, J. S., Cherepashchuk, A. M., and Efremov, Y. N. 1972, Nature, 236, 448.

Uitterdijk, F. 1932, B.A.N., 6, 295.

Wackerling, L. R. 1971, Mem. R.A.S., 73, 153.

Webster, B. L., and Murdin, P. 1972, Nature, 235, 37. 